\title{
Mudança tecnológica ou inércia: uma análise comparativa internacional com vistas ao caso brasileiro
}

\author{
Rosa Caiazza ${ }^{1}$ \\ Francisco Ebeling ${ }^{2}$ \\ Graziella Ferrara ${ }^{3}$
}

\section{RESUMO}

Tomando como ponto de partida a perspectiva neoinstitucionalista de que a dependência da trajetória afeta o curso de difusão de inovações tecnológicas, nós investigamos que fatores levam à mudança ou à inércia tecnológica. Perseguindo este objetivo, apresentamos um quadro geral capaz de identificar o papel dos policy-makers de afetar processos de difusão de inovações tecnológicas. Discutimos alguns casos que evidenciam a relação entre o modelo da dependência da trajetória determinística e o "development approach", respectivamente, com as experiências de alguns países de sucesso e fracasso na difusão tecnológica. Os resultados obtidos são debatidos à luz do caso brasileiro.

Palavras-chave: tecnologia; inovação; Brasil; policy makers.

\section{ABSTRACT}

Embracing neo-institutionalism's perspective that path dependence affects trajectories of innovation diffusion, we investigate factors that lead to technological change or inertia. At this aim, we first present a general framework able to identify the role of policy-makers in affecting innovation diffusion processes. We discuss some cases able to evidence the relation between the deterministic path dependence model and the development approach with success and failure in countries' technological diffusion. The results obtained are discussed in light of the Brazilian case.

Keywords: technology; innovation; Brazil; policy makers.

\section{INTRODUÇÃO}

Os estudos neoinstitucionalistas, especialmente os da escola histórica, abraçaram a perspectiva da dependência da trajetória (path dependence) a fim de explicar como as instituições afetam a dinâmica dos processos sociais (NORTH, 1990). Desde então, a dependência da trajetória tornou-se um conceito capaz de explicar como o passado molda o futuro também no âmbito dos estudos sobre a tecnologia e a inovação. Tomando como ponto de partida a perspectiva neoinstitucionalista de que a dependência da trajetória afeta as

\footnotetext{
${ }^{1}$ PhD. Professora da Università degli Studi di Napoli Parthenope (UniParthenope), Itália

${ }^{2}$ MSc., doutorando pelo Programa de Pós-Graduação em Políticas Públicas, Estratégias e Desenvolvimento da Universidade Federal do Rio de Janeiro (UFRJ). E-mail: ebelingbarros@ gmail.com

${ }^{3}$ Dra. Professora da Università degli Studi Suor Orsola Benincasa (UNISOB), Itália
} 
trajetórias de difusão de inovações, nós nos perguntamos quais fatores podem explicar a mudança ou a inércia tecnológica. Mais especificamente, os policy-makers podem apoiar a difusão de inovações através de políticas gerais, do lado da oferta e da demanda, por meio de medidas econômicas, jurídicas, estruturais e culturais (SURIÑACH et al., 2009).

Assim, combinamos modelos de difusão de tecnologia e tipos de políticas governamentais, de forma a oferecer um quadro geral para explicar o sucesso e o fracasso na difusão de uma nova tecnologia ou de uma inovação. Em seguida, apresentamos uma tipologia de países e/ou regiões, em que relacionamos o modelo de dependência da trajetória determinista e o "development approach" com, respectivamente, sucesso e fracasso na difusão de novas tecnologias. Assim, usamos o arcabouço teórico para discutir a Venezuela, a Alemanha, o Reino Unido e o Silicon Valley enquanto casos de sucesso ou fracasso.

$\mathrm{Na}$ seção I, apresentamos dois modelos de dependência da trajetória. Na seção II evidenciamos o papel dos policy-makers no fomento da mudança, descrevendo um conjunto de ferramentas modernas de medidas de política das quais um governo pode lançar mão a fim de orientar a mudança tecnológica. Na seção III, discutimos alguns casos de sucesso e fracasso. Na seção IV, contextualizamos os achados obtidos nas seções anteriores para o caso brasileiro. Finalmente, na seção V, apresentamos as conclusões.

\section{ARCABOUÇO TEÓRICO}

O modelo determinístico da dependência da trajetória evidencia que, quando uma tecnologia torna-se dominante através do seu uso repetido, a probabilidade de que as pessoas se desviem desse caminho declina progressivamente. Uma vez que uma massa crítica de indivíduos tenha adotado a tecnologia, um processo de feedback positivo vai estabilizar o que Ebbinghaus (2005) chama de "trodden trail" (caminho pisado), quando cada vez mais pessoas orientam as suas decisões com base na percepção de que um número suficiente de outras pessoas também o fizeram.

Algumas condições precisam ser preenchidas para que se caracterize o modelo da dependência da trajetória determinística (ARTHUR, 1994). Primeiro, assume-se uma condição de partida igual (probabilidade) para cada tecnologia existente no período $t_{1}$. Dentre as várias opções possíveis, a tecnologia que será a mais adotada pelos atores depende, durante as fases iniciais do processo de difusão tecnológica, do acaso. Segundo, são os mecanismos sociais de self-reinforcement, a partir da escolha de muitos, que tornam uma alternativa 
tecnológica a mais atraente. Assim, a difusão de uma tecnologia ocorre por meio da percepção da existência de externalidades de rede. Como consequência deste processo social de escolha, a adoção de uma tecnologia vai se estabilizar ao longo do tempo.

Para a continuidade do nosso argumento, é importante destacar que a dependência das trajetórias pode também explicar as características institucionais de um determinado país. Boyer (2005), por exemplo, argumenta que os países combinam em diferentes proporções os diversos mecanismos de coordenação existentes: para além dos Estados e dos mercados, também as comunidades, redes, associações e organizações privadas. As diferentes proporções em que os mecanismos de coordenação são combinados por cada país explicam, em certa medida - não desconsiderando outros fatores, tais como a dotação de recursos naturais, o que cada país se especializa em produzir.

Em contraste com a dependência das trajetórias determinística, a qual assume que eventos randômicos terão efeitos de longo prazo sobre a inércia tecnológica, o "developmental approach" - ou o que se pode chamar também de "dependência da trajetória nãodeterminística" (EBBINGHAUS, 2005) - centra-se na possibilidade de que atores sociais introduzam mudanças. Enquanto no primeiro modelo esta decorre, sobretudo, de choques exógenos, no segundo uma nova tecnologia pode tornar-se dominante em uma nova junção crítica, em que os atores coletivos - dos quais, na maioria dos casos, se sobressaem os atores estratégicos - fazem novas escolhas.

Em clave análoga, Crouch (2009) reconstrói a teoria da dependência das trajetórias equipando-a com a possibilidade de que ocorram mudanças-, a fim de propor o que ele chama de "governança recombinante". De acordo com este conceito, os empreendedores institucionais - ou, mais precisamente, os atores estratégicos - podem usar sua influência a fim de orientar a mudança institucional para novas direções, criando, assim, novas dependências da trajetória. Por isso, no "developmental approach", tecnologias podem tornar-se dominantes por causa de decisões conscientes tomadas por atores coletivos.

A seleção de uma via é, portanto, o resultado de uma escolha coletiva que é tomada em uma janela de oportunidades na qual algumas possíveis alternativas estão disponíveis (STINCHCOMBE, 1975). Decisões anteriores, uma vez institucionalizadas, estruturam as alternativas para as posteriores, mapeando os novos caminhos que poderão ser percorridos (ROKKAN, 1999). Neste ponto, atores coletivos decidem qual das tecnologias alternativas disponíveis preferem adotar. Assim, o processo subsequente de difusão depende de um processo de auto-reforço. Neste caso, os processos de auto-reforço de difusão da tecnologia 
anterior chegaram ao fim e as inovações se estabilizam. Assim, o feedback positivo permite a aceitação social de uma nova tecnologia.

\section{RESULTADOS E DISCUSSÃO}

\subsection{Políticas}

Os policy-makers podem influenciar a dependência da trajetória do processo de difusão tecnológica de diversas maneiras. Dessas decisões de política decorrem a mudança ou a inércia tecnológica.

Mais especificamente, os policy-makers podem influenciar, através de um conjunto de vários tipos de incentivos, a adoção de uma tecnologia ou inovação por uma massa crítica de pessoas, a despeito da existência de várias outras trajetórias tecnológicas disponíveis. A isso chamamos, conforme a figura 1 abaixo, de "processo de seleção da tecnologia", que, assim cabe ressaltar, também pode ocorrer quando uma determinada rota tecnológica é escolhida sem a interferência dos governos. Assim, entre novas e múltiplas possíveis opções, a escolha social de continuar a adotar uma tecnologia existente é afetada pelas decisões de política.

Em algum ponto no tempo, um governo pode perceber que é necessário induzir a mudança para outra trajetória tecnológica. Tendo em mãos um conjunto de políticas, este pode apoiar a adoção de uma nova tecnologia. Neste caso, os formuladores de política que têm a influência e o poder político para dirigir a mudança institucional para novas direções, criam, portanto, novas dependências da trajetória.

Assim, uma nova tecnologia pode tornar-se dominante por causa de escolhas conscientes dos policy-makers. Decisões anteriores, uma vez institucionalizadas, estruturam as alternativas para as posteriores, criando um novo caminho. Neste ponto, atores coletivos decidem qual das tecnologias alternativas disponíveis preferem adotar. Assim, o processo subsequente de difusão depende de um processo de auto-reforço. Neste caso, os processos de auto-reforço de difusão da tecnologia anterior chegou ao fim e as inovações são estabelecidas em seu lugar. Assim, o feedback positivo permite a aceitação social de uma tecnologia recém lançada. 
Figura 1 - O processo de difusão tecnológica

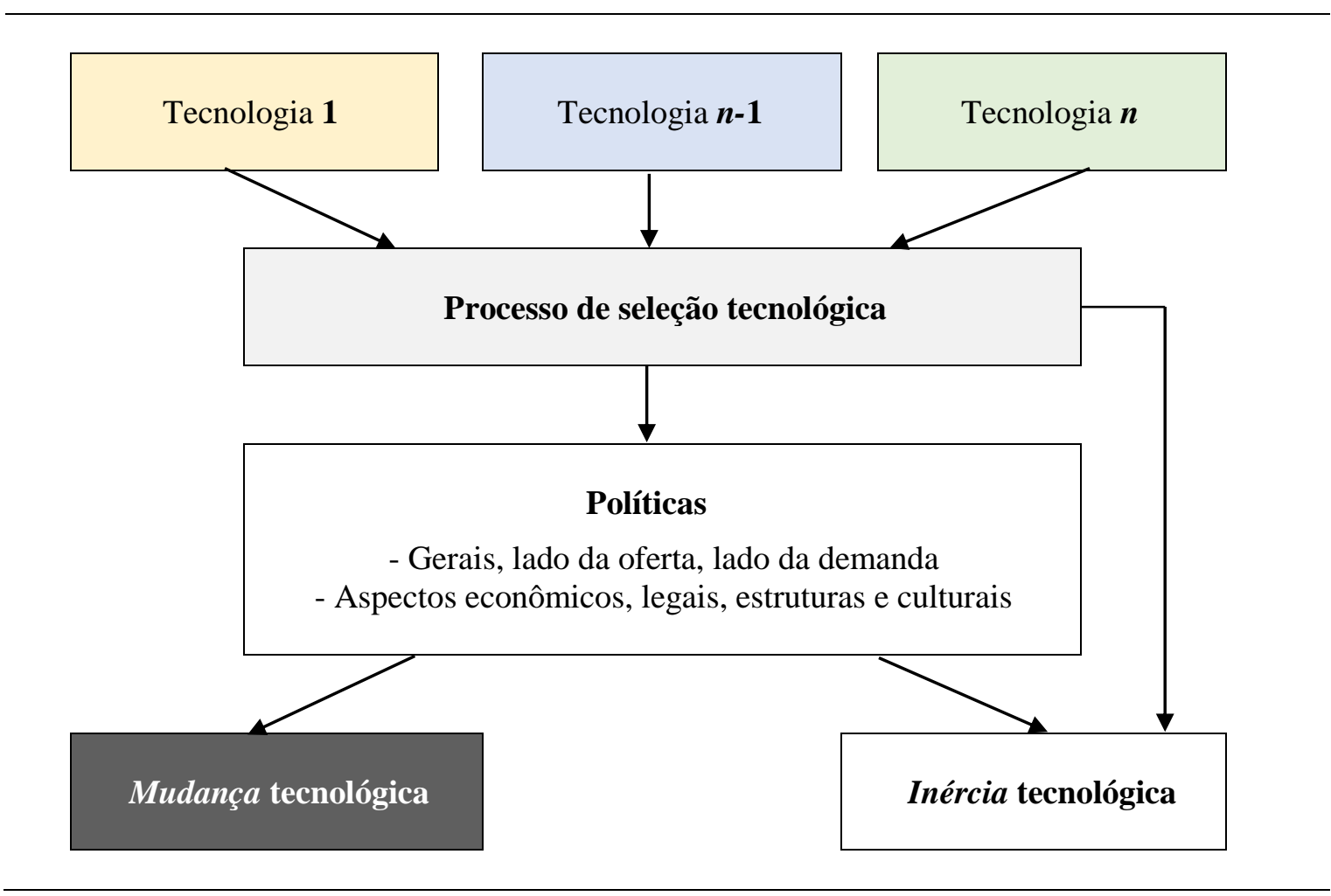

Fonte: Elaboração própria

Como mencionado acima, perseguindo o objetivo de afetar o processo social de difusão de uma tecnologia específica, os policy-makers podem adotar tanto políticas gerais, como pelo da oferta e do lado da demanda que, por sua vez, possuem aspectos econômicos, legais, estruturais e culturais.

Políticas gerais visam atualizar e regulamentar o quadro econômico em que as atividades inovadoras ocorrem. Políticas do lado da oferta são instrumentos destinados a fornecer inovadores com fundos, recursos humanos e assistência técnica de forma a enfrentar a resistência por parte do mercado em relação à difusão da inovação. Políticas pelo lado da demanda geralmente aparecem sob a forma de aumentos da demanda agregada que, direta ou indiretamente, estimulam o florescimento de atividades inovadoras. Na definição dessas políticas, os governos podem influenciar aspectos econômicos, legais, estruturais e culturais, que facilitam a difusão de uma tecnologia, ao invés de outra (EDLER 2007, 2008).

Especificamente, os policy-makers podem focar sua atenção em aspectos econômicos e jurídicos, adotando medidas macroeconômicas, tais como incentivos fiscais ou financeiros 
e programas de depreciação favoráveis às empresas que investem em novas tecnologias. Os formuladores de políticas podem promover a difusão de uma tecnologia desenvolvendo a normalização, que gerará convergência entre os inovadores e aqueles que adotam a tecnologia, no que concerne padrões e compatibilidade tecnológicos (STONEMAN, 1989; BINGHAM, 1978; MILES E SNOW, 1978). Os policy-makers podem também apoiar os inovadores absorvendo parte dos custos financeiros em que estes incorrem quando promovem inovações, bem como apoiá-los em sua estratégia de internacionalização. Um governo pode também patrocinar o risco que os usuários enfrentam ao selecionar e adotar uma nova tecnologia, assim como tornar-se o principal comprador de uma inovação (BOZEMAN et al., 1978). Os governos podem também adotar políticas que reduzam as restrições financeiras enfrentadas pelos usuários - associadas aos custos iniciais ou ao longo do processo de adoção da nova tecnologia-, tais como: ajuda financeira direta, subsídios, empréstimos, e taxas de juros subsidiadas. Estes podem, ademais, adotar as novas tecnologias para melhorar a qualidade dos bens e serviços públicos, reduzindo custos, dessa forma indo de encontro às novas demandas da sociedade (BROWN et al., 1990).

Os policy-makers podem adotar medidas estruturais destinadas a integrar as estratégias de vários atores (DAMANPOUR E GOPALAKRISHNAN, 2001). Eles podem apoiar os inovadores que criam centros de pesquisa onde se promove o upgrade tecnológico. Estes podem, ademais, estender as capacidades de centros de pesquisa já existentes, facilitando as iniciativas lideradas pela indústria e apoiando a adaptação da tecnologia às necessidades do mercado (GEROSKI, 1990). Os policy-makers também podem criar centros públicos que sensibilizem os potenciais usuários sobre as vantagens das novas tecnologias disponíveis, sobre suas possíveis aplicações e seus benefícios. Outra possibilidade é também criar centros de tecnologia aplicada com pessoal capaz de oferecer assistência técnica, consultoria tecnológica, além de realizar avaliações e recomendações para as empresas em indústrias locais (RUSSELL E RUSSELL, 1992).

Finalmente, os formuladores de políticas podem atuar sobre os aspectos culturais, através de uma série de medidas destinadas a facilitar a colaboração entre as empresas: ao reduzir os riscos associados com o desenvolvimento de novos produtos; ao facilitar a resolução de problemas comuns, a partilha de informação e de aprendizagem, a obtenção de economias de escala, o fortalecimento de negócios em andamento, e as relações de desenvolvimento de tecnologia (HALL E KHAN, 2003). Os níveis de comunicação e de diálogo entre os desenvolvedores de tecnologia e usuários, bem como entre os usuários, a 
credibilidade institucional e a liderança, dentre outros aspectos relativos ao capital social e cultural, têm se mostrado extremamente importantes e eficazes para a criação de uma propensão cultural para a difusão de tecnologia (HALL, 2004; MAHAJAN E PETERSON, 1985).

Nesse sentido, o Governo pode fornecer apoio para a realização de parcerias públicoprivadas, destinadas a desenvolver e comercializar a nova tecnologia e evidenciar novas oportunidades, em termos de mercado, aos usuários potenciais (KARAGOZOGLU, 1993; Lua e BRETSCHNEIDER, 1997). Ao mesmo tempo, os tomadores de decisão podem apoiar programas que divulguem informações com o objetivo de reduzir, sob a ótica dos usuários, os custos relacionados à busca da nova tecnologia; podem criar programas de intercâmbio, visando aperfeiçoar a capacidade de absorção das novas tecnologias por parte dos usuários, ou até mesmo a capacidade dos seus funcionários de absorver as novas tecnologias.

Os programas de treinamento, tais como a formação profissional, formação em sala de aula, seminários de gestão, oficinas de construção de equipe, e ensino à distância também são possibilidades (ROLFSTAM, 2005). Eles também podem apoiar a experimentação de uma nova tecnologia, proporcionando programas de informação sobre a sua existência e seus benefícios, centros de assistência técnica que facilitem a transição entre tecnologias, programas de treinamento para uso, e ações de demonstração para mostrar os benefícios da adoção (VON HIPPEL, 1976).

\subsection{Casos}

$\mathrm{Na}$ presente seção, apresentaremos uma tipologia de países e/ou regiões em que relacionamos o modelo da dependência da trajetória determinística e o "development approach" com, respectivamente, casos de sucesso e de fracasso na difusão de novas tecnologias, tais como estes aparecem na literatura. A tabela 1 abaixo sintetiza os casos apresentados:

Tabela 1 - Casos

\begin{tabular}{l|c|c}
\hline & $\begin{array}{c}\text { Dependência da } \\
\text { trajetória determinística }\end{array}$ & Development Approach \\
\hline Fracasso & Venezuela & Reino Unido \\
\hline Sucesso & Alemanha & Vale do Silício \\
\hline
\end{tabular}




\section{Fonte: Elaboração própria}

O exemplo que relaciona a dependência da trajetória determinística com o fracasso na difusão tecnológica é o da Venezuela. Em seu trabalho clássico, Karl (1997) explica que o petróleo molda a estrutura dos interesses organizados e o próprio Estado. Quando um 'petroestado' é um grande receptor de receitas decorrentes da venda do petróleo nos mercados internacionais e, ao mesmo tempo, não possui instituições maduras, existe um terreno fértil para a ação dos chamados 'rent-seekers'.

O resultado mais provável é, então, a chamada "maldição dos recursos", quando um país não é capaz de transformar sua riqueza mineral em bem-estar generalizado (Ebeling, 2014). Sabe-se que até a presidência de Hugo Chávez, que assumiu o cargo em 1998, as receitas do petróleo venezuelano foram em grande parte desperdiçadas. No entanto, Chávez começou a usar essas receitas a fim de melhorar os indicadores sociais, sobretudo em saúde e educação, tendo alcançado grande sucesso. Muito rapidamente, a Venezuela logrou construir capital cultural, que pode levar a mudanças no futuro. Contudo, a Venezuela ainda é economicamente frágil, uma vez que ainda não conseguiu diversificar significativamente as suas atividades econômicas não relacionadas à extração de petróleo.

Uma interpretação para isso pode ser encontrada em Medeiros (2012). Ele argumenta que os Estados que foram capazes de construir, em um momento inicial, uma base industrial razoavelmente sólida, têm maiores chances de escapar da "maldição dos recursos naturais". A Venezuela, ao contrário da Rússia, nunca conseguiu fazer isso na história da sua indústria de petróleo. Quando os preços do petróleo caem abruptamente, tal como ocorre durante o curso da escrita deste artigo, o orçamento público fica seriamente constrangido, o que diminui ainda mais a possibilidade de que as políticas que apoiam a difusão de inovações tecnológicas acima aludidas - sejam deslanchadas.

Nós agora relacionamos a dependência da trajetória determinística com um caso de sucesso, com o exemplo da Alemanha. A fim de explicar o caso alemão, recorremos a Linda Weiss e à literatura das variedades de capitalismo (VOC), de Hall e Soskice (2009). Desde o século 19, argumenta Weiss (1998), o estado alemão possui capacidades transformativas e distributivas que lhe permitiram construir uma base industrial muito sólida e um estado de bem-estar social muito consistente. Até então, os objetivos do país eram realizar o "catching up" com os seus competidores - notadamente a Inglaterra - e construir uma economia de guerra. 
Por isso, desde o início a Alemanha tomou uma série de medidas para alavancar a sua indústria, que se tornou conhecida internacionalmente por sua qualidade e robustez. Um exemplo disso é o sucesso da Alemanha em transformar em realidade a assim chamada "Energiewende", onde as fontes alternativas de energia estão sendo introduzidas na rede em um ritmo muito rápido.

Talvez o sucesso da Energiewende possa ser explicado por uma combinação de medidas, tais como: incentivos fiscais e suporte financeiro, desenvolvimento de instituições de pesquisa de alto nível, como os institutos Max-Planck, e de uma força de trabalho muito bem treinada. Não obstante, mais recentemente, a literatura das variedades de capitalismo, que compara economias de mercado liberais (Liberal Market Economies - LMEs) - onde os EUA é o caso paradigmático - com economias de mercado coordenadas (Coordinated Market Economies - CMEs) - onde o caso paradigmático é a Alemanha - afirma que, enquanto a primeira se destaca em inovações radicais, a última se sobressai em inovações incrementais.

Assim, empresas como a Daimler Benz e a Siemens, embora sejam reconhecidas internacionalmente pela qualidade de seus produtos, não teriam a capacidade de trazer para o mercado produtos e serviços radicalmente diferentes. Em suma, afirmar-se-ia que a indústria da Alemanha estaria condenada a ter certo padrão de difusão tecnológica, em que as inovações não divergiriam significativamente do padrão atual. É improvável que ocorra uma mudança radical neste padrão porque as despesas da Alemanha em infraestrutura e investimentos sociais - que incluem P,D\&I - foram drasticamente reduzidas (STREECK E MERTENS, 2013).

Agora relacionamos o "development approach" com o fracasso, citando os casos interligados da Inglaterra e da Escócia, em um exemplo de uma mudança da trajetória que não levam a um novo padrão de difusão tecnológica. O Reino Unido foi o primeiro país a se industrializar completamente (CUMBERS, 2012, p. 226), e a cidade de Londres sempre foi um centro financeiro muito importante. Ao redor de Londres - ao menos até os anos 1970-, a Grã-Bretanha logrou implementar um complexo híbrido entre Keynesianismo e uma abordagem mais do tipo laissez-faire.

O primeiro concentrou-se na produção de bens nas indústrias produtivas, bem como na entrega de serviços públicos que capacitassem os indivíduos para exercer funções para além da esfera financeira. $\mathrm{Na}$ década de 1970, quando os parâmetros do sistema keynesiano tornaram-se cada vez mais difíceis de serem sustentados, a City também vivia um dos seus momentos mais difíceis. Na década de 1980, uma série de inovações importantes na esfera 
financeira impulsionou a cidade para ter uma nova importância global, especialmente com a liberalização dos movimentos de capitais (CROUCH, 2009, p. 148).

Assim, o modelo keynesiano foi abandonado, sendo substituído por estruturas neoliberais. Isso criou uma nova dependência da trajetória. Some-se a isso que, nos anos 1970 e 1980, os policy-makers optaram por canalizar as receitas da produção de petróleo obtida no Mar do Norte escocês para reforçar políticas macroeconômicas de coorte monetarista, a fim de impulsionar ainda mais a city de Londres como um centro financeiro, e para fornecer um ambiente "amigável e eficiente" para as empresas estrangeiras com as competências técnicas adequadas para desenvolver, o mais rápido possível, os recursos do Mar do Norte, ao invés de perseguir uma estratégia industrial intervencionista (CUMBERS, 2012, p. 229).

No que diz respeito à uma possível política de inovação que poderia ter introduzido a um novo padrão de difusão tecnológica, Mazzucatto (2011) explica que, nas últimas três décadas, a Grã-Bretanha "fez tudo errado", com implicações negativas para o crescimento de longo prazo. Ela explica que os impostos dos contribuintes são mal direcionados e que as oportunidades estão sendo perdidas. Uma política de inovação, em sua opinião, precisaria concentrar-se na criação de condições que permitam que as inovações floresçam, tanto através de políticas pelo lado da demanda como pelo lado da oferta, bem como através da produção de inovações por parte do próprio Estado. Muito embora Londres seja um hub poderoso, em que os inovadores de todo o mundo podem se conectar e ter acesso a diversas formas de financiamento, e em que a criação do conhecimento tem encontrado quase que um "habitat natural", pouco tem sido feito nas últimas décadas pelo governo para apoiar a inovação tecnológica.

Em suma, os policy-makers britânicos concentram-se demasiadamente em políticas do tipo laissez faire, que podem ter bons resultados em certos contextos, mas que também relegam regiões inteiras do Reino a um grande atraso econômico e social.

Para concluir, relacionamos agora o "development approach" com o sucesso extremo, citando o exemplo do Vale do Silício. Mazzucatto (2013) argumenta que, desde a década de 1970, este se tornou o ' $h u b$ ' norte-americano de inovações no âmbito da microinformática, devido a uma série de parcerias público-privadas e ao papel de liderança do governo no financiamento e pesquisa (básica e aplicada).

Esta combinação de fatores gestou oportunidades que foram muito bem aproveitadas por empresários inovadores no chamado "Silicon Gold Rush". O que poderia ser chamado de "Estado desenvolvimentista norte-americano", cuja existência poucos reconhecem, sempre 
ofereceu um kit completo de ferramentas para apoiar a difusão tecnológica, em todos os domínios apresentados na seção anterior. Mas, talvez a medida mais importante tomada pelo "Estado desenvolvimentista norte-americano" para apoiar o seu desenvolvimento industrial, considerando que as atividades empreendidas no Vale do Silício envolviam riscos consideráveis, eram do tipo econômico e financeiro, com as autoridades governamentais encomendando diretamente aos fornecedores e destes adquirindo soluções inovadoras, especialmente para fins de sua economia de guerra.

O que distingue a experiência no Vale do Silício de todas as outras que se observam no mundo é que uma recessão como o estouro da bolha "dot-com", no início de 2000, pôde ser facilmente revertida. Nesse sentido, Mazzucatto (2013) mostra que a criação do Iphone só foi possível porque a Apple conseguiu combinar astutamente uma série de tecnologias que o ' $h u b$ ' norte-americano de inovações no âmbito da microinformática e o "Estado desenvolvimentista norte-americano" haviam gestado, tais como:

“(i) microprocessor or central processing units (CPU); (2) dynamic random-access memory (DRAM); as well as (3) micro hard drive storage or hard drive disks (HDD); (4) liquidcrystal displays (LCDs); (5) lithium-polymer (Li-Pol) and lithium-ion (Li-ion) batteries; (6) digital signal processing (DSP), based on the advanced in fast Fourier transform (FFT) algorithms; (7) the Internet; (8) the Hypertext Transfer Protocol (HTTP) and Hypertext Markup Language (HTML); (9) (...) cellular technology and networks (...); (10) global positioning systems (GPS); (11) click-wheel navigation and multi-touch screens; (12) and artificial intelligence with a voice-user interface program" (MAZZUCATTO, 2013, p. 95).

Em suma, o Vale do Silício é um exemplo de um hub ou um cluster de inovação em que a criação e a combinação de novas tecnologias praticamente não conhecem limites, e em que a intervenção do Estado em alguns casos, já não se revela necessária. Do nosso ponto de vista, este é o exemplo mais claro de uma combinação do "development approach" com a contínua e bem sucedida difusão tecnológica.

\subsection{O caso do Brasil}

Do nosso ponto de vista, o caso do Brasil é distintivo por que ele apresenta, em alguma medida, de forma estilizada, características de todos os casos acima mencionados.

Da Venezuela, o Brasil toma a iminência de uma possível maldição dos recursos naturais, sobretudo em estados como o Rio de Janeiro, muito dependentes da produção de petróleo e de sua cadeia industrial - onde são adquiridos bens e serviços necessários para a extração petrolífera. 
Entretanto, e, justamente em função da existência dessa cadeia, o país tem a chance de criar uma nova dependência da trajetória, rumo a uma indústria que seja, como um todo, mais diversificada e densa tecnologicamente. Ademais, o país aposta no petróleo como uma fonte de receitas para o aperfeiçoamento de suas políticas educacional e de saúde, sobretudo por meio da nova lei dos royalties (Lei $\mathrm{n}^{\circ} 12.858$ ), segundo a qual $75 \%$ dos royalties dos campos cuja produção se iniciar em 2013 devem obrigatoriamente ser destinados à educação e 50\% dos retornos do Fundo Social à educação e à saúde.

Da Alemanha, o Brasil toma a baixa propensão à inovação radical de alguns setores consolidados de sua indústria, como, por exemplo, os setores automobilístico e petrolífero, ainda muito dependentes da importação de tecnologia estrangeira.

Do Reino Unido, tomamos o consenso neoliberal em torno do 'tripé macroeconômico', que torna praticamente compulsórias elevadas taxas de juros para conter a inflação. Estas, por sua vez, comprimem bastante o orçamento público que, em outro cenário, poderiam privilegiar muito mais as políticas educacionais, de inovação, industrial, etc.

Dos Estados Unidos, tomamos a experiência de alguns clusters ou hubs industriais e de inovação, tal como o aeronáutico - ao redor da Embraer, em São José dos Campos-, e o da Ilha do Fundão - onde, além do CENPES, se instalaram uma série de centros de pesquisa empresariais do setor petrolífero. Estes clusters ou hubs, entretanto, não se comparam com o Vale do Silício, seja em robustez ou em capacidade inovadora e/ou tecnológica.

A partir do quadro analítico que apresentamos neste artigo, para que o país consiga inaugurar uma nova dependência da trajetória, mais calcada em inovação, é evidente que os policy-makers brasileiros deverão lançar mão de políticas gerais, pelo da oferta e do lado da demanda, em seus aspectos econômicos, legais, estruturais e culturais.

Em suma, estamos falando da implementação de uma política de desenvolvimento produtiva suficientemente ampla e compreensiva, que reconheça a complexidade da política industrial, e que contemple e compreenda as complexas inter-relações existentes entre os níveis macro, meso e micro, conforme a figura 2 a seguir.

Para dirigir a mudança institucional para novas direções e, portanto, criar novas dependências da trajetória, é preciso que os formuladores de política tenham bastante influência e o poder político adequado, algo que não parece se avizinhar no atual cenário econômico e político brasileiro. 
Figura 2 - A relação entre os inputs, outcomes e os níveis de coordenação ao longo dos vários níveis da política industrial

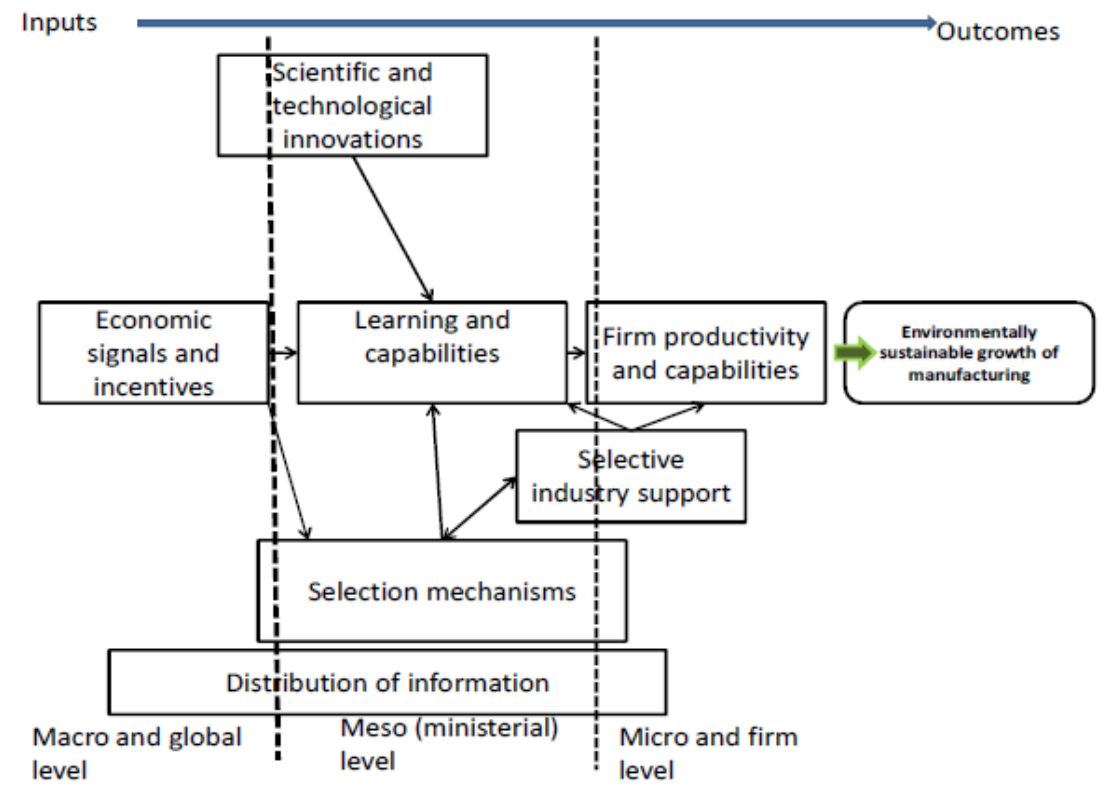

Fonte: Naudé, 2010

\section{CONCLUSÕES}

Tendo tomado como ponto de partida a perspectiva neoinstitucionalista de que a dependência da trajetória afeta processos de difusão da inovação, o objetivo deste trabalho foi investigar que fatores levam à mudança ou à inércia tecnológica.

Perseguindo este objetivo, na seção 1, apresentamos dois modelos concorrentes ambos calcados na teoria da dependência da trajetória - que explicam os possíveis caminhos percorridos pela difusão de uma inovação. O primeiro modelo salienta que a difusão de uma inovação em um momento $t_{1}$, e seu uso continuado nos períodos subsequentes, provavelmente resulta em inércia tecnológica. A segunda visão evidencia que a combinação de vários eventos leva a mudanças de tecnologia. Na seção 2, evidenciamos o papel que, em ambos os casos, os formuladores de políticas podem assumir para influenciar difusão social de uma tecnologia em detrimento de outra. Assim, combinando diferentes modelos de dependência das trajetórias, que explicam ou não a difusão tecnológica, com possíveis políticas governamentais, sugerimos um quadro geral para explicar o sucesso ou fracasso da difusão de uma nova tecnologia. Na seção 2 objetivamos também fornecer uma análise moderna do que 
os países em desenvolvimento podem fazer a fim de orientar a mudança tecnológica para novas direções.

Na seção 3, apresentamos uma tipologia de países e/ou regiões em que relacionamos, respectivamente, o modelo de dependência das trajetórias determinista e o "development approach" com sucesso e fracasso na difusão de novas tecnologias. Os países e/ou regiões discutidos como caso de sucesso ou fracasso foram: Venezuela, Alemanha, Inglaterra e Silicon Valley (EUA).

Antes de chegar ao término deste artigo, cabem algumas observações finais, que podem até mesmo estender este debate para outros trabalhos. Embora tenhamos mostrado alguns casos que, atualmente, se encaixam muito bem nos modelos que apresentamos, devese reconhecer que, rigorosamente falando, o termo "dependência das trajetórias" nada mais é do que um sinônimo de "a história importa“, como discutido por Boldizzonni (2011). Embora este seja um termo forte - ou uma "grade narrativa" para explicar por que as instituições de alguns países aparecem em uma determinada maneira ou por que certos países produzem o que produzem, deve ser considerado que a história tem uma dinâmica própria, que nem sempre se encaixa em modelos ou pré-concepções. Isso é o que Streeck e Thelen (2009) tinham em mente quando argumentaram que a mudança institucional é um processo dinâmico, interminável, e muitas vezes sutil. Por sua vez, Pierson (2004) argumentou que uma característica importante da dependência da trajetória é o que timing relativo importa; isto é, a probabilidade de um "early comer" ser o caminho escolhido é maior do que a de um retardatário. No entanto, no mesmo livro, ele também havia afirmado que, no longuíssimo prazo instituições podem mudar consideravelmente. Em suma, a obsolescência é uma característica que, com grande facilidade, pode ser inerente a muitas instituições, de tal maneira que os casos que nós apresentamos neste artigo podem ter um desempenho bastante diverso no futuro.

Além disso, há de se ressaltar também que há um debate quase infinito se o que mais importa para o desenvolvimento socioeconômico são as instituições ou a acumulação de capital. Nesse sentido, Glyn escreve que:

\footnotetext{
"a acumulação de capital é a força motriz fundamental da economia. Aumentos de investimento são geralmente o elemento mais dinâmico em expansões de demanda agregada, particularmente em escala mundial, onde as exportações de um país são as importações do outro. (...) O investimento tem uma relação simbiótica com a introdução de novas tecnologias "(GLYN, 2007, p. 86, tradução livre).
} 
Nesse sentido, nada garante que um aumento maciço da demanda agregada na Venezuela - o caso que apresentamos como exemplo de "lock-in" de uma dada trajetória-, por exemplo, através de investimentos feitos pelo recém-fundado banco dos BRICS, possa alterar todo esse quadro de relativa penúria econômica. Principalmente considerando que, através da intervenção do governo Hugo Chávez, o país agora possui um nível educacional bastante considerável.

Para concluir, quando discutimos as rotas que a difusão tecnológica pode tomar, devemos ter muito cuidado com a questão da dotação de recursos, ou seja, com as chamadas "vantagens comparativas". Embora estas possam explicar de forma muito fiel algumas estratégias que alguns países perseguem - já que a concorrência é muito forte na escala mundial-, é importante lembrar que, como Stiglitz (2012) argumenta, "o que importa é a vantagem comparativa dinâmica, ou a vantagem comparativa a longo prazo, que pode ser moldada". Portanto, um conselho para os países em desenvolvimento é que as políticas para apoiar a difusão de tecnologia - econômica e jurídico, estrutural e cultural - sejam estas do tipo geral, pelo lado da oferta ou pelo lado da demanda, nunca devem ser abandonadas. Como argumenta Heise (2007), as políticas monetárias, fiscal ou salarial não logram atingir o crescimento sustentado e o pleno emprego de forma isolada, o que significa que as diversas políticas - inclusive as macroeconômicas - são interdependentes e devem ser coordenadas. Contra a orientação hayekiana de que a intervenção é mais danosa do que útil, a implementação de políticas macroeconômicas, industriais, etc, é quase sempre desejável, a despeito de sua viabilidade ser 'constrangida' (HEISE, 2008). As restrições internas e/ou externas que se impõem à implementação dessas políticas, apesar de imporem enormes desafios aos países, podem ser superadas através da ação política coletiva, como mostra Evans (2006, 2008).

\section{REFERÊNCIAS}

ARTHUR, W. B., Increasing Returns and Path Dependence in the Economy. Ann Arbor, MI:University of Michigan Press, 1994.

BINGHAM, R. D., Innovation, bureaucracy, and public policy: A study of innovation adoption by local government, The Western Political Quarterly, 31(2), 178-205, 1978.

BOLDIZZONI, F. The Poverty of Clio: ressurrecting economic history. Princeton: Princeton University Press, 2011.

BOYER, R. How and Why Capitalisms Differ. MPIfG Discussion Paper 05/4, 2005. Disponível em: 〈http://www.mpifg.de/pu/dp09-13_de.asp>. Acesso em: 20 ago. 2014. 
BOZEMAN B., ROERING K., SLUSHER E. Social Structures and the Flow of Scientific Information in Public Agencies: An Ideal Design, Research Policy 7(4), 384-405, 1978.

BROWN M.A., BERRY L., GOEL R., Guidelines for Successfully Transferring Governmentsponsored Innovations, Research Policy 20(2), 121-143, 1990.

CROUCH, C. Capitalist Diversity and Change: Recombinant Governance and Institutional Entrepreneurs. New York: Oxford, 2009

DAMANPOUR F., GOPALAKRISHNAN S., Dynamics of the adoption of product and process innovations in organizations. Journal of Management Studies 28, 45-65, 2001.

EBBInghaUS, B., Can Path Dependence Explain Institutional Change? Two Approaches Applied to Welfare State Reform. MPIfG Discussion Paper 05/2. Germany, 2005.

EBELING, F., Can Oil Steer Brazil's Social and Economic Development? An Alternative New Institutionalist Approach. The Journal of Energy and Development, vol 39, n. 2, 171206, 2014.

EDLER J. Demand-based innovation policy, Manchester Business School Working Paper, N. 529, 2007.

EDLER J. The role of demand-side policies in innovation policy, presentation to the OECD Working Party on Innovation and Technology Policy, 2008.

EDLER J., GEORGHIOU L. Public procurement and innovation. Resurrecting the demand side, Research Policy, 36(1), 949-963, 2007.

EDQUiST C., HOMMEN L., TSIPOURI L., Public Technology Procurement and Innovation, Springer, 2000.

EVANS, P. Extending the 'Institutional Turn'. World Institute for Development Economics Research Research Paper n. 113, Outubro de 2006. University Press, 2009.

EVANS, P. In Search of the 21st Century Developmental State. The Centre for Global Political Economy. University of Sussex. UK. Working Paper No 4, 2008.

GEROSKI P.A., Procurement policy as a tool of industrial policy, International Review of Applied Economics, Vol. 4 (2), 182-198, 1990.

GLYN, A. Capitalism Unleashed. Oxford: Oxford University Press, 2007.

HALL B. H. Innovation and diffusion, NBER Working Paper Series. Working Paper n 10212, 2004.

HALL B., KHAN B. Adoption of New Technology, In Jones, Derek C., New Economy Handbook, Amsterdam: Elsevier Science, 2003.

HALL, P.; SOSKICE, D. An Introduction to Varieties of Capitalism. In. HANCKÉ, B. (org). Debating Varieties of Capitalism. New York: Oxford University Press, 2009. p. 21- 74

HEISE, A. Institutions, market constellations and growth: The case of South Africa. Annals of Economic and Finance, 2007, 8-2, 313-340 
A Post Keynesian theory of economic policy - filling a void, Arbeitspapiere für Staatswissenschaft, No. 28, 2008

KARAGOZOGLU N., (1993) Environmental uncertainty, strategic planning, and tecnica competitive advantage, Technovation 13(6), 335-347.

KARL, T. L. The Paradox of Plenty: Oil Booms and Petro-States. Berkeley: University of California Press, 1997.

MAHAJAN V., Peterson R.A. Models of Innovation Diffusion, Beverly Hills, Sage Publications, 1985.

MAZZUCATO, M. The entrepreneurial state. London: Anthem Press, 2013.

MEDEIROS, C. A. Natural Resources Nationalism and Development Strategies. Paper presented at the 2012 ESHET Conference, St. Petersburg, May, 17-19, 2012

MILES R.E., SNOW C.C. (1978), Organizational strategy, structure, and process. McGraw-Hill, NewYork, 1978.

MOON M. J., BRETSCHNEIDER S. Can State Government Actions Affect Innovation and Its Diffusion?: An Extended Communication Model and Empirical Test. Technological Forecasting and Social Change 54(1):57-77. 1997.

NAUDÉ, W. Industrial Policy: Old and New Issues. UNU Wider Working Paper No. 2010/106, september 2010.

NORTH, D.C., Institutions, Institutional Change and Economic Performance. Cambridge: Cambridge University, 1990.

PIERSON, P. Placing politics in time. Princeton: Princeton University Press, 2004.

ROKKAN, S., State Formation, Nation-Building and Mass Politics in Europe. In: FLORA P., KUHNLE S., URWIN D. (ed.), The Theory of Stein Rokkan, Oxford:Oxford University Press, 1999.

ROLFSTAM M. Public Technology Procurement as a Demand-side Innovation Policy Instrument: An Overview of Recent Literature and Events Division of Innovation. Lund Institute of Technology, Lund University, 2005.

RUSSELL R., RUSSELL C., An examination of the effects of organizational norms, organizational structure, and environmental uncertainty on entrepreneurial strategy. Journal of Management 18(1), 639-657, 1992.

STIGLITZ, J.E. From Resource Curse to Blessing. Project Syndicate, 6/08/2012. Available at: <http://www.projectsyndicate.org/commentary/from-resource-curse-to-blessing-by-josephe--stiglitz>

STINCHCOMBE, A.L., Social Structure and Politics. In: FRED I.G., NELSON W.P. (eds.), Macropolitical Theory, Reading MA:Addisson-Wesley, 1975.

STONEMAN P., Technological diffusion and vertical product differentiation, Economics Letters, 31(3), 277-280, 1989. 
STREECK, W., MERTENS, D. Public Finance and the Decline of State Capacity in Democratic Capitalism. In. SCHÄFER, A., STREECK, W. Politics in the Age of Austerity. Cambridge: Polity Press, 2012.

STREECK, W.; THELEN, K. Institutional Change in Advanced Political Economies. In. Hancké, B. (org). Debating Varieties of Capitalism. New York: Oxford University Press 2009. p. 95-131.

SURIÑACH J., et al., The Diffusion/Adoption of Innovation in the Internal Market, European Commission Economic Papers 384 September, 2009.

VON HIPPEL, E., The dominant role of users in the scientific instrument innovation process, Research Policy, 5(3), 212-239, 1976.

WEISS, L. The myth of the powerless state. Ithaca: Cornell University Press, 1998. 